\title{
Numerical Applications of Conformal Mapping with Explicit Formulae Based on the Power Series Method
}

\author{
Yaocai MA ${ }^{\mathrm{a}}$, Aizhong LU ${ }^{\mathrm{a}, 1}$, Hui CAI ${ }^{\mathrm{a}}$, Xiangtai $\mathrm{ZENG}^{\mathrm{b}}$ \\ anstitute of Hydroelectric and Geotechnical Engineering, North China Electric Power \\ University, Beijing 102206, China \\ ${ }^{\mathrm{b}}$ School of Civil Engineering and Architecture, Nanchang University, Nanchang, \\ 330031, China
}

\begin{abstract}
A general optimization method, based on the power series method, is presented for computing the conformal mappings with explicit expressions from: (a) the unit disc onto an infinite domain exterior of a closed Jordan curve, (b) the circular annulus domain onto a finite doubly-connected domain bounded by two closed Jordan curves, (c) the infinite domain bounded by two circular curves onto an infinite domain bounded by two non-circular closed Jordan curves. The unknown mapping functions are approximated by the power series method. The problem of solving the mapping function coefficients is transformed into the problem of determining the image points on the image plane by means of the least square method. Different from most of the previous optimization methods, the angles are set as the design variables rather than the mapping function coefficients in the paper. The influence of the terms of the series on the calculation accuracy is investigated. The successful applications of the proposed method are shown by a large number of numerical examples.
\end{abstract}

Keywords. Conformal mapping, Jordan curve, power series method, least square method, optimization method

\section{Introduction}

The conformal mapping, which can transform the physical regions with complex geometry onto simpler computational regions, is a fundamental tool in complex analysis. It has many successful applications in heat conduction, electromagnetics, electrostatics, hydrodynamics, aerodynamics and grid generation [1-2]. Especially, the conformal mapping is of great significance in the stress analysis of structural components with various shaped holes in 2D elasticity theory, which requires an explicit expression of mapping function. Therefore, an effective and accurate method for determining the mapping function is crucial for both theoretical signification and practical application. There are two types of conformal mappings: the mapping from a problem region to a socalled canonical region, and the inverse mapping from a canonical region to a problem

\footnotetext{
${ }^{1}$ Corresponding Author, Aizhong LU, Institute of Hydroelectric and Geotechnical Engineering, North China Electric Power University, Beijing 102206, China; Email: lvaizhong@ncepu.edu.cn.
} 
region. The problem of finding the conformal mappings has attracted the interest of many researchers, who approached the problem by using many methods.

The conformal mapping of the interior or the exterior of the unit circle to a given domain bounded by a closed Jordan curve has been well researched. One popular method involves the integral equation methods (e.g., Mikhlin's integral, Symm's integral and Theodorsen's integral), which can be reduced to the derivation and solution of the integral equations for the boundary correspondence. The Fast Fourier Transform algorithm, orthonormal polynomials method, Wegmann's method and Lagrange's interpolation method can be introduced to solve the equations [3]. Particularly, the Schwarz-Christoffel transformation [4-6] were applied to solve mappings that involve regions with polygonal boundaries. Papamichael and Kokkinos [7] used the Bergman kernel method and the Ritz method to solve the mapping. The mapping function of the interior or exterior the unit circle can be approximately described by a truncated Taylor series. Fornberg [8] utilized the Fourier transform method and Lu and Wang [9] used the optimization method to determine the leading Taylor coefficients. Some iterative methods are also proposed to approach the problem, such as the method of searching mapping points on the boundary $J[10]$ and the method based on the triangle interpolation theory [11].

The problem of finding the conformal mapping of a circular annulus onto a finite doubly connected region with two Jordan curves is also important in conformal transformation. Many solutions [12-15] are confined to the regions whose boundaries are circular or axisymmetric with respect to coordinate axes. Vecheslavov and Tolstobrova [16] developed the Schwarz-Christoffel transformation for doubly connected polygonal domains. In particular, Papamichael and Kokkinos [17] used the singular functions to solve the mapping problem of domains with sharp corners. For a more general domain, the integral equation method presented by Symm [18], the iterative method developed by Wegmann [19] and the conjugate function method used by Hakula et al. [20] can be introduced.

There are some methods for obtaining the conformal mapping of multiply connected domains with connectivity larger than two. Mayo [21] employed Mikhlin's integral equation to determine the mapping function which maps multiply connected regions onto slit discs. Perhaps the ideal canonical domains may be circular since they can bring a lot of convenience in application. The Schwarz-Christoffel transformation for conformal mappings from circular domains onto polygonal domains were presented by DeLillo et al. [22], Crowdy [23-24] and DeLillo [25]. Zeng and Lu [26] considered the conformal mapping which maps two circular domains onto an infinite domain bounded by two arbitrarily shaped curves to solve the twin-tunnel problem in underground engineering. A new general form of mapping function in the form of power series was proposed and the optimization method was applied to determine the mapping function coefficients. But the solution is confined to the case where the curves are symmetric with respect to $\mathrm{x}$ axis. The general Schwarz-Christoffel integral for multiply connected domains was derived by Mityushev [27-28].

In summary, the integral method has the advantage of obtaining the conformal transformation of domains with complicated boundaries. The power series method has also been used for solving simply and multiply connected domain problem [29-31]. Although there are some approximations since the power series are adopted, the proposed method can provide explicit expressions and determine the mapping function coefficients, which is urgently needed in the stress analysis of structural components and 
underground constructions. The application of the power series method in mechanics was systematically developed by Savin [32].

This paper proposed a general method for computing the conformal mapping functions. The unknown forms of the mapping functions are described by power series. The method ingeniously sets the angles on the image plane as the quantities to be solved rather than the mapping function coefficients by using the least square principle. Then the problem is transformed into an optimization problem with the angles as design variables, which makes it easy to evaluate the initial values of the design variables in the range of $[0,2 \pi]$. In addition, the method has no restrictions on the shape and symmetry of the computational domains on the z-plane. The mixed penalty function method is used as an optimization tool to solve the problem effectively. Numerical examples collected from various structural components and underground engineering are given to prove that the method has successful applications in the following conformal maps:

(a) The mapping from the unit disc onto a domain exterior of a closed Jordan curve (figure 1).

(b) The mapping from the annulus domain onto a finite doubly-connected domain bounded by two closed Jordan curves (figure 2).

(c) The mapping from an infinite domain bounded by two circular curves onto an infinite domain bounded by two arbitrary closed Jordan curves (figure 3 ).

\section{Mapping Functions Described by Power Series}

\subsection{The Mapping of an Infinite Domain Bounded by a Closed Jordan Curve}

Let $D_{z}^{a}$ be an infinite domain exterior of the Jordan curve $J_{a}$ in the $z$-plane (figure 1). The conformal mapping function from the unit disc $D_{\zeta}^{a}=\{\zeta:|\zeta| \leq 1\}$ in the $\zeta$-plane onto $D_{z}^{a}$ can be approximated by a truncated Taylor series as follows [1].

$$
z=\omega_{a}(\zeta)=\sum_{k=-1}^{n} c_{k} \zeta^{k}
$$

where $\zeta=\rho e^{i \theta}, i$ is the imaginary unit, and the coefficients $c_{k}(k=-1,0,1, \ldots, n)$ are all complex numbers for a general domain.

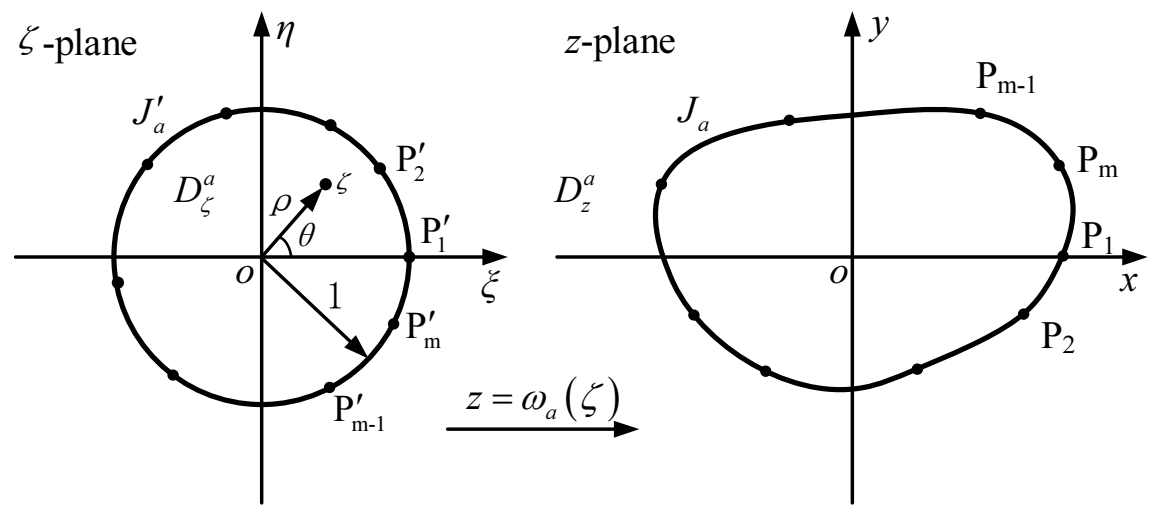

Figure 1. The conformal mapping from the unit disc onto the infinite domain exterior of an arbitrary closed Jordan curve. 


\subsection{The Mapping of an Annular Domain Bounded by Two Closed Jordan Curve}

As shown in figure $2, D_{z}^{b}$ is a finite doubly-connected domain in the $z$-plane, bounded externally and internally by two closed Jordan curves $J_{b 1}$ and $J_{b 2}$, respectively. Consider the problem of conformally mapping a circular annulus domain $D_{\zeta}^{b}=\left\{\zeta: r_{b} \leq|\zeta| \leq 1\right\}$ in the $\zeta$-plane onto $D_{z}^{b}$ in such a way that $J_{b 1}$ corresponds to the inner, and $J_{b 2}$ to the outer circumference. The ratio of the radii of the annulus domain is unique [18]. Suppose the outer radius is 1 and the inner radius is $r_{b}\left(0<r_{b}<1\right)$. Of course, $r_{b}$ is initially unknown and has to be determined. The mapping function can be approximated by a truncated Laurent series.

$$
z=\omega_{b}(\zeta)=\sum_{k=-l}^{n} c_{k} \zeta^{k}
$$

where the coefficients $c_{k}(k=-l,-(l-1), \ldots,-1,0,1, \ldots,(n-1), n)$ are all complex numbers for a general domain.

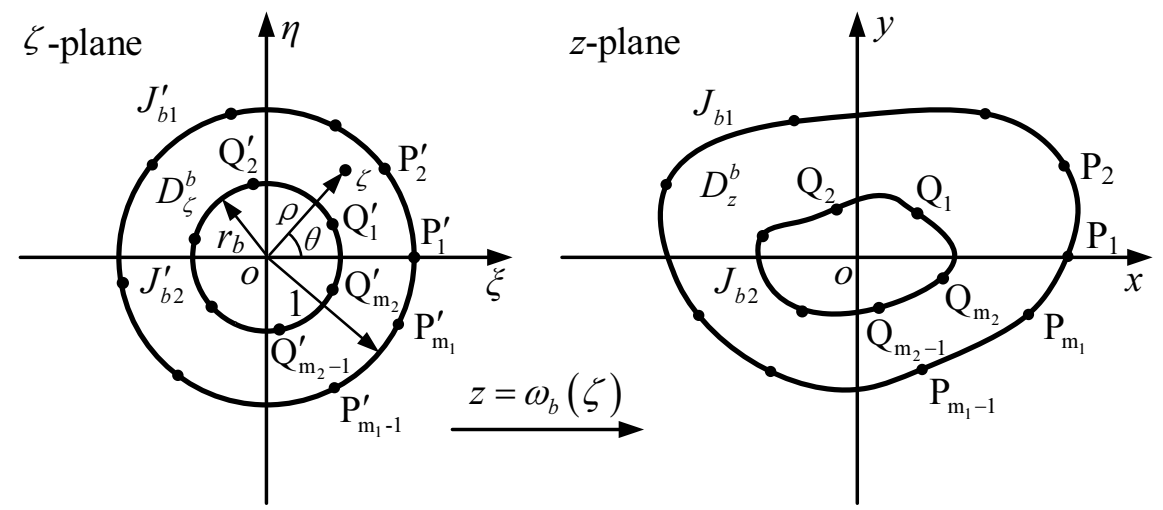

Figure 2. The conformal mapping from a circular annulus domain onto the finite domain bounded by two arbitrary closed Jordan curves.

\subsection{The Mapping of an Infinite Domain Bounded by Two Closed Jordan Curves}

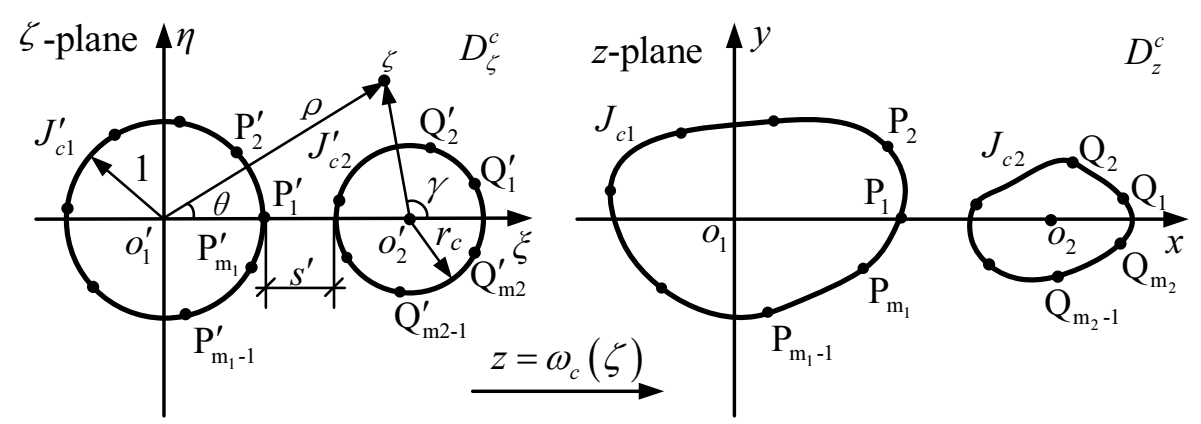

Figure 3. The conformal mapping from an infinite domain bounded by two circular curves onto the infinite domain bounded by two arbitrary closed Jordan curves.

Let $D_{z}^{c}$ be a triply-connected domain in the $z$-plane, bounded by two non-circular closed Jordan curves $J_{c 1}$ and $J_{c 2}$. Zeng and $\mathrm{Lu}$ [26] found the conformal mapping function, which maps the infinite domain $D_{\zeta}^{c}$ (figure 3 ) bounded by two circular curves onto $D_{Z}^{c}$. 
The left circle and right circle in the $\zeta$-plane correspond to $J_{c 1}$ and $J_{c 2}$, respectively. The radius of the left circle is fixed as 1 . The radius of the right circle is indicated as $r_{c}$ and remains to be solved. And the mapping function has the following general form

$$
z=\omega_{c}(\zeta)=c^{\prime} \zeta+c_{0}+\sum_{k=1}^{n_{1}} c_{k} \zeta^{-k}+\sum_{k=1}^{n_{2}} c_{-k}(\zeta-S)^{-k}
$$

where $s^{\prime}$ is the distance between the two circular curves in the $\zeta$-plane, $S=1+s^{\prime}+r_{c}$ and the coefficients $c^{\prime}, c_{0}, c_{k}\left(k=1,2, \ldots, n_{1}\right)$ and $c_{-k}\left(k=1,2, \ldots, n_{2}\right)$ are all complex numbers for a general domain.

\section{Solution of Mapping Function Coefficients}

In the section, the general method for solving the mapping function coefficients in $\omega_{a}(\zeta)$, $\omega_{b}(\zeta), \omega_{c}(\zeta)$ is presented. Although the forms of the functions are different, the mapping problem can be transformed into an optimization problem.

\subsection{Coefficients in $\omega_{a}(\zeta)$}

On the $J_{a}^{\prime}$ (figure 1 ), we have $\rho=1$, and $\zeta=e^{i \theta}$. Assume that $m$ mapping points are provided clockwise in the $z$-plane to describe the boundary $J_{a}$. Let $z_{j}=x_{j}+$ $i y_{j}(j=1,2, \ldots, m)$ be a point on the $J_{a}$, corresponding to $\zeta_{j}=e^{i \theta_{j}}$ on the $J_{a}^{\prime}$. The $\theta_{j}(j=1,2, \ldots, m)$ are unknown when the mapping function coefficients are not given. $\mathrm{P}_{1}$ fixed at the $x$-axis corresponds to $\mathrm{P}_{1}^{\prime}$ fixed at the $\xi$-axis. Then the following equation is obtained according to Eq. (1).

$$
z_{j}=\omega_{a}\left(\zeta_{j}\right)=\sum_{k=-1}^{n} c_{k} \zeta_{j}^{k}=\sum_{k=-1}^{n} c_{k} e^{i k \theta_{j}}, j=1,2, \ldots, m
$$

Eq. (4) can be written as

$$
\mathbf{Z}=\mathbf{X Y}
$$

where

$$
\begin{gathered}
\mathbf{Z}=\left[\begin{array}{lllll}
z_{1} & z_{2} & \cdots & z_{m}
\end{array}\right]^{\mathrm{T}}, \\
\mathbf{X}=\left[\begin{array}{cccccc}
\zeta_{1}^{-1} & 1 & \zeta_{1}^{1} & \zeta_{1}^{2} & \cdots & \zeta_{1}^{n} \\
\zeta_{2}^{-1} & 1 & \zeta_{2}^{1} & \zeta_{2}^{2} & \cdots & \zeta_{2}^{n} \\
\vdots & \vdots & \vdots & \vdots & \vdots & \vdots \\
\zeta_{m}^{-1} & 1 & \zeta_{m}^{1} & \zeta_{m}^{2} & \cdots & \zeta_{m}^{n}
\end{array}\right] \text { and } \\
\mathbf{Y}=\left[\begin{array}{lllll}
c_{-1} & c_{0} & c_{1} & \cdots & c_{n}
\end{array}\right]^{\mathrm{T}} .
\end{gathered}
$$

Once $\mathbf{X}$ is given, $\mathbf{Y}$ can be evaluated by the complex least-square method [33], we have

$$
\hat{\mathbf{Y}}=\left(\overline{\mathbf{X}}^{\mathrm{T}} \mathbf{X}\right)^{-1} \overline{\mathbf{X}}^{\mathrm{T}} \mathbf{Z}
$$

where $\overline{\mathbf{X}}$ means to carry out conjugate operation for each element in the matrix $\mathbf{X}$, and $\widehat{\mathbf{Y}}$ is the estimate of $\mathbf{Y}$ that allows

$$
e=(\hat{\mathbf{Z}}-\mathbf{Z})^{\mathrm{T}}(\hat{\mathbf{Z}}-\mathbf{Z})
$$


to reach the minimum value, where $\widehat{\mathbf{Z}}=\mathbf{X} \widehat{\mathbf{Y}}$.

It can be observed that Eq. (6) will be reduced to the solution of a set of complex linear equations if $m=n+1$, i.e., $\widehat{\mathbf{Y}}=\mathbf{X}^{-1} \mathbf{Z}$. In order to achieve high accuracy, this paper takes $m>n+1$. This means that Eq. (4) will be satisfied exactly if the error $e$ (Eq. (7)) is equal to zero. Our problem then is to find approximate solution $\theta^{*}=$ $\left(\theta_{1}, \theta_{2}, \ldots, \theta_{m}\right)$ (corresponding to $\mathrm{X}^{*}$ ) that minimizes $e$. The problem can be solved by means of optimization technique. The mixed penalty function method [34], which can solve optimization problems with equality and inequality constraints, is introduced herein to address the mapping function coefficients. The $\theta_{j}(j=1,2, \ldots, m)$ are set as the design variables. Eq. (7) is the objective function. Evidently, we have

$$
\theta_{1}=0, \theta_{1}<\theta_{2}<\ldots<\theta_{m}<2 \pi
$$

Therefore, the following constraints are made:

$$
\left\{\begin{array}{l}
\theta_{1}=0 \\
\theta_{j+1}-\theta_{j}>0(j=1,2, \ldots, m-1) \\
2 \pi-\theta_{m}>0
\end{array}\right.
$$

There are 1 equality constraint and $m$ inequality constraints. Once $\theta^{*}$ is obtained, the mapping function coefficients $\mathbf{Y}^{*}$ can be given by

$$
\mathbf{Y}^{*}=\left({\overline{\mathbf{X}^{*}}}^{\mathrm{T}} \mathbf{X}^{*}\right)^{-1} \overline{\mathbf{X}^{*}} \mathbf{Z}
$$

In theory, the mapping function coefficients will be real numbers if the given domain is symmetric with respect to $x$-axis. To obtain the accurate real mapping function coefficients, the following steps are performed. First we have

$$
\mathbf{U}=\mathbf{V Y}
$$

where $\mathbf{U}=\left[\begin{array}{c}\operatorname{Re}(\mathbf{Z}) \\ \operatorname{Im}(\mathbf{Z})\end{array}\right]$ and $\mathbf{V}=\left[\begin{array}{c}\operatorname{Re}(\mathbf{X}) \\ \operatorname{Im}(\mathbf{X})\end{array}\right]$. The signs $\operatorname{Re}()$ and $\operatorname{Im}()$ mean to find the real part and imaginary part of each element of the matrix in bracket. Then, $\mathbf{Y}$ can be evaluated by the real least square method presented by Gauss.

$$
\hat{\mathbf{Y}}=\left(\mathbf{V}^{\mathrm{T}} \mathbf{V}\right)^{-1} \mathbf{V}^{\mathrm{T}} \mathbf{U}
$$

The objective function is

$$
e=(\hat{\mathbf{U}}-\mathbf{U})^{\mathrm{T}}(\hat{\mathbf{U}}-\mathbf{U})
$$

where $\widehat{\mathbf{U}}=\mathbf{V} \widehat{\mathbf{Y}}$. And the equality and inequality constraints remain unchanged. Similarly, $\mathbf{Y}^{*}$ can be given by

$$
\mathbf{Y}^{*}=\left(\mathbf{V}^{* \mathrm{~T}} \mathbf{V}^{*}\right)^{-1} \mathbf{V}^{* \mathrm{~T}} \mathbf{U}
$$

where $\mathrm{V}^{*}$ corresponds to the optimal solution $\theta^{*}$.

\subsection{Coefficients in $\omega_{b}(\zeta)$}

In figure $2, m_{1}$ and $m_{2}$ mapping points are provided anticlockwise to describe the boundaries $J_{b 1}$ and $J_{b 2}$, respectively. $\mathrm{P}_{1}$ fixed at the $x$-axis corresponds to $\mathrm{P}_{1}^{\prime}$ fixed at the $\xi$-axis. Let $z_{j}=x_{j}+y_{j}$ be a point on the $J_{b 1}$ (corresponding to $j=1,2, \ldots, m_{1}$ ) or $J_{b 2}$ (corresponding to $j=m_{1}+1, m_{1}+2, \ldots, m_{1}+m_{2}$ ). The points on the $J_{b 1}^{\prime}$ and $J_{b 2}^{\prime}$ are 
$\zeta_{j}=e^{i \theta_{j}}\left(j=1,2, \ldots, m_{1}\right) \quad$ and $\quad \zeta_{j}=r_{b} e^{i \theta_{j}}\left(j=m_{1}+1, m_{1}+2, \ldots, m_{1}+m_{2}\right)$, respectively. Then the following equation is obtained according to Eq. (2).

$$
z_{j}=\omega_{b}\left(\zeta_{j}\right)=\sum_{k=-l}^{n} c_{k} \zeta_{j}^{k}=\left\{\begin{array}{l}
\sum_{k=-l}^{n} c_{k} e^{i k \theta_{j}}, j=1,2, \cdots, m_{1} \\
\sum_{k=-l}^{n} c_{k} r_{b}^{k} e^{i k \theta_{j}}, j=m_{1}+1, m_{1}+2, \cdots, m_{1}+m_{2}
\end{array}\right.
$$

Let $m=m_{1}+m_{2}$, then Eq. (14) can be written in the form of Eq. (5), the difference is that

$$
\begin{aligned}
\mathbf{X} & =\left[\begin{array}{ccccccccc}
\zeta_{1}^{-l} & \zeta_{1}^{-(l-1)} & \ldots & \zeta_{1}^{-1} & 1 & \zeta_{1} & \cdots & \zeta_{1}^{(n-1)} & \zeta_{1}^{n} \\
\zeta_{2}^{-l} & \zeta_{2}^{-(l-1)} & \ldots & \zeta_{2}^{-1} & 1 & \zeta_{2} & \cdots & \zeta_{2}^{(n-1)} & \zeta_{2}^{n} \\
\vdots & \vdots & \ldots & \vdots & \vdots & \vdots & \cdots & \vdots & \vdots \\
\zeta_{m}^{-l} & \zeta_{m}^{-(l-1)} & \ldots & \zeta_{m}^{-1} & 1 & \zeta_{m} & \cdots & \zeta_{m}^{(n-1)} & \zeta_{m}^{n}
\end{array}\right] \text { and } \\
\mathbf{Y} & =\left[\begin{array}{lllllllll}
c_{-l} & c_{-(l-1)} & \ldots & c_{-1} & c_{0} & c_{1} & \ldots & c_{n-1} & c_{n}
\end{array}\right]^{\mathrm{T}}
\end{aligned}
$$

at this time.

The objective function is the same as Eq. (7). The $\theta_{j}$ and $r_{b}$ are set as the design variables. And there are 1 equality constraint and $m+2$ inequality constraints.

$$
\left\{\begin{array}{l}
r_{b}>0 \\
1-r_{b}>0 \\
\theta_{1}=0 \\
\theta_{j+1}-\theta_{j}>0\left(j=1,2, \cdots, m_{1}-1\right) \\
2 \pi-\theta_{m_{1}}>0 \\
\theta_{j+1}-\theta_{j}>0\left(j=m_{1}+1,2, \cdots, m_{1}+m_{2}-1\right) \\
2 \pi-\theta_{m}>0
\end{array}\right.
$$

\subsection{Coefficients in $\omega_{c}(\zeta)$}

As can be seen in figure $3, m_{1}$ and $m_{2}$ control points are selected anticlockwise to describe the boundaries $J_{c 1}$ and $J_{c 2}$, respectively. $\mathrm{P}_{1}$ fixed at the $x$-axis corresponds to $\mathrm{P}_{1}^{\prime}$ fixed at the $\xi$-axis. Similarly, let $z_{j}=x_{j}+y_{j}$ be a point on the $J_{c 1}$ (corresponding to $j=1,2, \ldots, m_{1}$ ) or $J_{c 2}$ (corresponding to $j=m_{1}+1, m_{1}+2, \ldots, m_{1}+m_{2}$ ). The points on the $J_{c 1}^{\prime}$ are $\zeta_{j}=e^{i \theta_{j}}\left(j=1,2, \ldots, m_{1}\right)$. Herein, we introduce the unknown $\gamma$ to indicate the angle with $O_{2}^{\prime}$ as the vertex. Then, the points on the $J_{c 2}^{\prime}$ are $\zeta_{j+m_{1}}=S+$ $r_{c} e^{i \gamma_{j}}\left(j=1,2, \ldots, m_{2}\right)$. Then the following equation is obtained according to Eq. (3). 


$$
\begin{aligned}
& z_{j}=\omega_{c}\left(\zeta_{j}\right)=c^{\prime} \zeta_{j}+c_{0}+\sum_{k=1}^{n_{1}} c_{k} \zeta_{j}^{-k}+\sum_{k=1}^{n_{2}} c_{-k}\left(\zeta_{j}-S\right)^{-k} \\
& =\left\{\begin{array}{l}
c^{\prime} e^{\mathrm{i} \theta_{j}}+c_{0}+\sum_{k=1}^{n_{1}} c_{k} e^{-k \mathrm{i} \theta_{j}}+\sum_{k=1}^{n_{2}} c_{-k}\left(e^{\mathrm{i} \theta_{j}}-S\right)^{-k}, j=1,2, \cdots, m_{1} \\
c^{\prime}\left(S+r_{c} e^{\mathrm{i} \gamma_{j}}\right)+c_{0}+\sum_{k=1}^{n_{1}} c_{k}\left(S+r_{c} e^{\mathrm{i} \gamma_{j}}\right)^{-k}+\sum_{k=1}^{n_{2}} c_{-k} r_{c}^{-k} e^{-k \mathrm{i} \gamma_{j}}, j=1,2, \cdots, m_{2}
\end{array}\right.
\end{aligned}
$$
that

Let $m=m_{1}+m_{2}$, Eq. (16) can be written in the form of Eq. (5), the difference is

$$
\begin{gathered}
\mathbf{X}=\left[\begin{array}{cccccccccc}
\zeta_{1} & 1 & \zeta_{1}^{-1} & \zeta_{1}^{-2} & \cdots & \zeta_{1}^{-n_{1}} & \left(\zeta_{1}-S\right)^{-1} & \left(\zeta_{1}-S\right)^{-2} & \cdots & \left(\zeta_{1}-S\right)^{-n_{2}} \\
\zeta_{2} & 1 & \zeta_{2}^{-1} & \zeta_{2}^{-2} & \cdots & \zeta_{2}^{-n_{1}} & \left(\zeta_{2}-S\right)^{-1} & \left(\zeta_{2}-S\right)^{-2} & \cdots & \left(\zeta_{2}-S\right)^{-n_{2}} \\
\vdots & \vdots & \vdots & \vdots & \vdots & \vdots & \vdots & \vdots & \vdots & \vdots \\
\zeta_{m} & 1 & \zeta_{m}^{-1} & \zeta_{m}^{-2} & & \zeta_{m}^{-n_{1}} & \left(\zeta_{m}-S\right)^{-1} & \left(\zeta_{m}-S\right)^{-2} & \cdots & \left(\zeta_{m}-S\right)^{-n_{2}}
\end{array}\right] \text { and } \\
\mathbf{Y}=\left[\begin{array}{llllllllll}
c^{\prime} & c_{0} & c_{1} & c_{2} & \cdots & c_{n_{1}} & c_{-1} & c_{-2} & \cdots & c_{-n_{2}}
\end{array}\right]^{\mathrm{T}}
\end{gathered}
$$

at this time.

The objective function is the same as Eq. (7). The $\theta_{j}, \gamma_{j}, s^{\prime}$ and $r_{b}$ are set as the design variables. And there are 1 equality constraint and $m+2$ inequality constraints.

$$
\left\{\begin{array}{l}
r_{c}>0 \\
1-r_{c}>0 \\
\theta_{1}=0 \\
\theta_{j+1}-\theta_{j}>0\left(j=1,2, \cdots, m_{1}-1\right) \\
2 \pi-\theta_{m_{1}}>0 \\
\gamma_{j+1}-\gamma_{j}>0\left(j=1,2, \cdots, m_{2}-1\right) \\
2 \pi-\gamma_{m_{2}}>0
\end{array}\right.
$$

\section{Investigation on the Calculation Accuracy}

In Section 3, $m$ mapping points are provided to describe the corresponding boundaries. The more mapping points are provided, the closer the boundary is to the given shape. And the number of the design variables for solving $\omega_{a}(\zeta), \omega_{b}(\zeta)$ and $\omega_{c}(\zeta)$ are $m, m+$ 1 and $m+2$, respectively. However, as is known, the optimization algorithm might be invalid if the number of the design variables is too large. This paper suggests that the maximum number of the design variables should not exceed 80 in most cases. Then, the influence of the number of the terms in the mapping function on the calculation accuracy is investigated in the following section. 


\subsection{The Infinite Domain Bounded by a Square}

Let $D_{z}$ be the infinite domain which is bounded internally by a square with four corners at $z= \pm 1 \pm 1 i$. We select 32 mapping points on the square, that is, $m=32$. The mapping function $\omega_{a}(\zeta)$ can be computed by the method proposed in Section 3.1. The mapping points and the curves plotted by the obtained $\omega_{a}(\zeta)$ with different terms of $\zeta$ are shown in figure 4 . The errors are listed in table 1.

Table 1. The errors for different terms of a square.

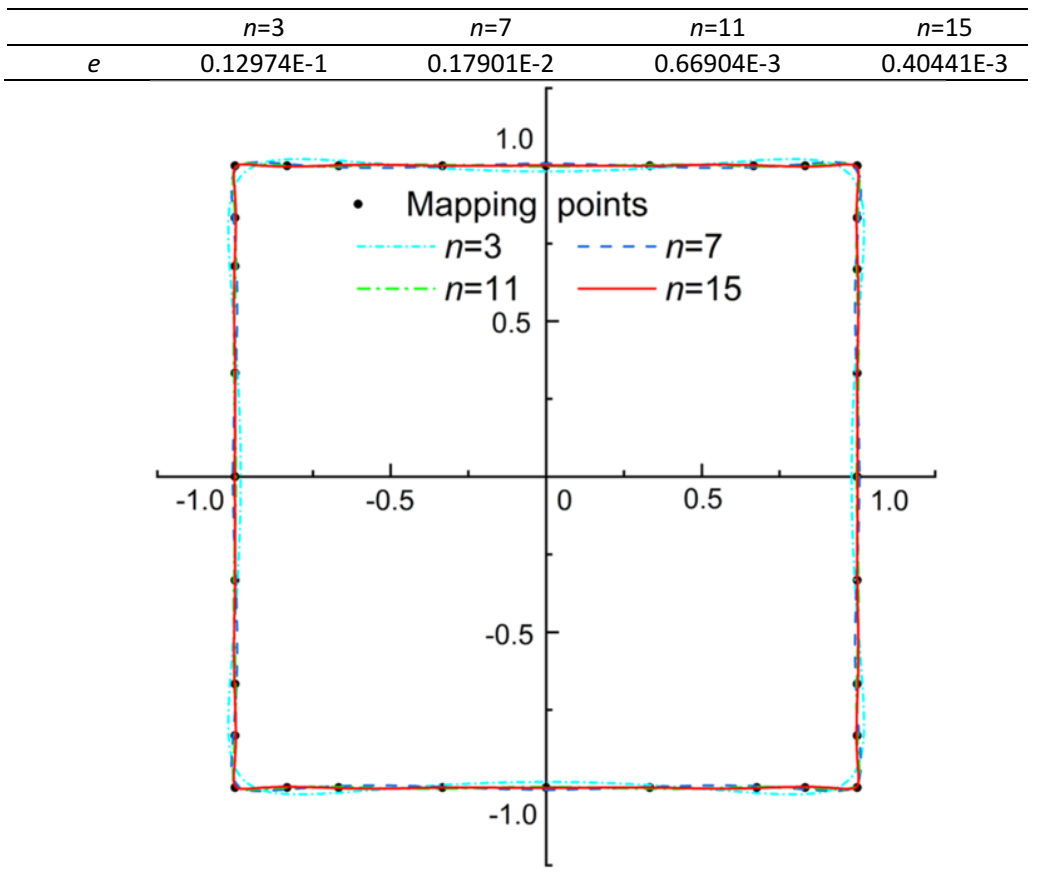

Figure 4. The mapping points and the curves plotted by different terms.

Table 1 shows that the error $e$ decreases as $n$ increases, which means that the solution will achieve high accuracy as long as the number of the terms of the mapping function is large enough. As can be seen in figure 4, the solution in this paper have high accuracy for larger $n$, even for the sharp corners. And it will be further demonstrated by a large number of numerical examples in the Section 5.

\subsection{The Annular Domain Bounded by a Square and a Circle}

Let $D_{z}$ be the finite domain which is bounded externally by a circle and internally by a square. The radius of the circle is 2 and the corners of the square are at $z= \pm 1 \pm 1 i .32$ mapping points are selected on each boundary, that is, $m=64$. Then the method presented in Section 3.2 are used to solve the mapping function coefficients for different positive and negative terms of $\zeta$ in $\omega_{b}(\zeta)$. The orthogonal curves on the $\zeta$-plane and $z$ plane are shown in figure 5 . The errors for different terms are listed in table 2. 
Table 2. The errors for different terms of a square in a circle.

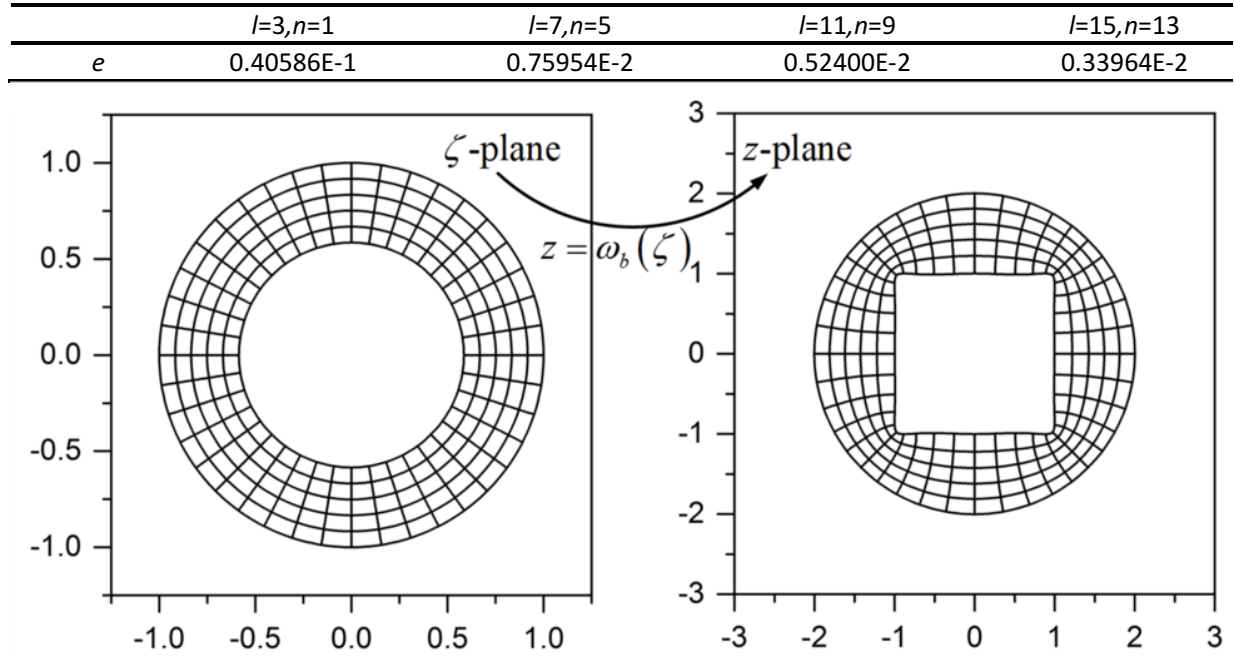

Figure 5. The conformal mapping from the annulus domain onto the finite domain bounded externally by a circle and internally by a square.

\subsection{The Infinite Domain Bounded by a Square and an Oval}

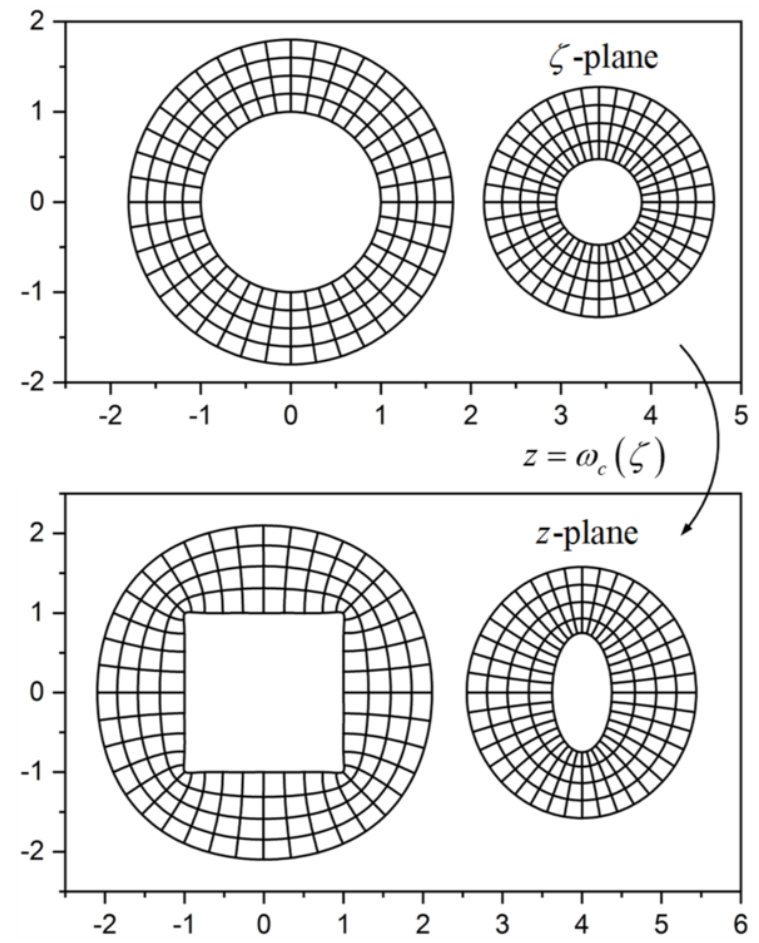

Figure 6. The conformal mapping from the circular domain onto the infinite domain bounded by a circle and an oval. 
Let $D_{z}$ be the infinite domain bounded by a square and an oval. The corners of the square are at $z= \pm 1 \pm 1 i$. The lengths of the major semi-axis and the minor semi-axis of the oval are 0.75 and 0.375 , respectively. Herein, we select 28 mapping points on each boundary, that is, $m=56$. Let $n_{1}=n_{2}$ in $\omega_{c}(\zeta)$. Then the influence of $n_{1}$ and $n_{2}$ on the accuracy is discussed. Table 3 lists the errors for different terms of $\omega_{c}(\zeta)$ computed by the method in Section 3.3. Figure 6 plots the orthogonal curves on the $\zeta$-plane and $z$ plane.

Table 3. The errors for different terms of a square and an oval.

\begin{tabular}{ccccc}
\hline & $n_{1}=n_{2}=5$ & $n_{1}=n_{2}=7$ & $n_{1}=n_{2}=9$ & $n_{1}=n_{2}=11$ \\
\hline$e$ & $0.70052 \mathrm{E}-2$ & $0.18630 \mathrm{E}-3$ & $0.16263 \mathrm{E}-3$ & $0.48704 \mathrm{E}-4$ \\
\hline
\end{tabular}

\section{Numerical Examples}

\subsection{The Infinite Domain Bounded By a Closed Jordan Curve}

This section mainly investigated the given domains which are not symmetric with respect to the axes of the coordinates. Consider the infinite domains $D_{z}$ bounded internally by an oval, a triangle, a square and a hexagon, respectively (figure 7). The mapping function coefficients are listed in table 4 . Notice that the numbers in bracket represent the real and imaginary parts of the coefficients, respectively.
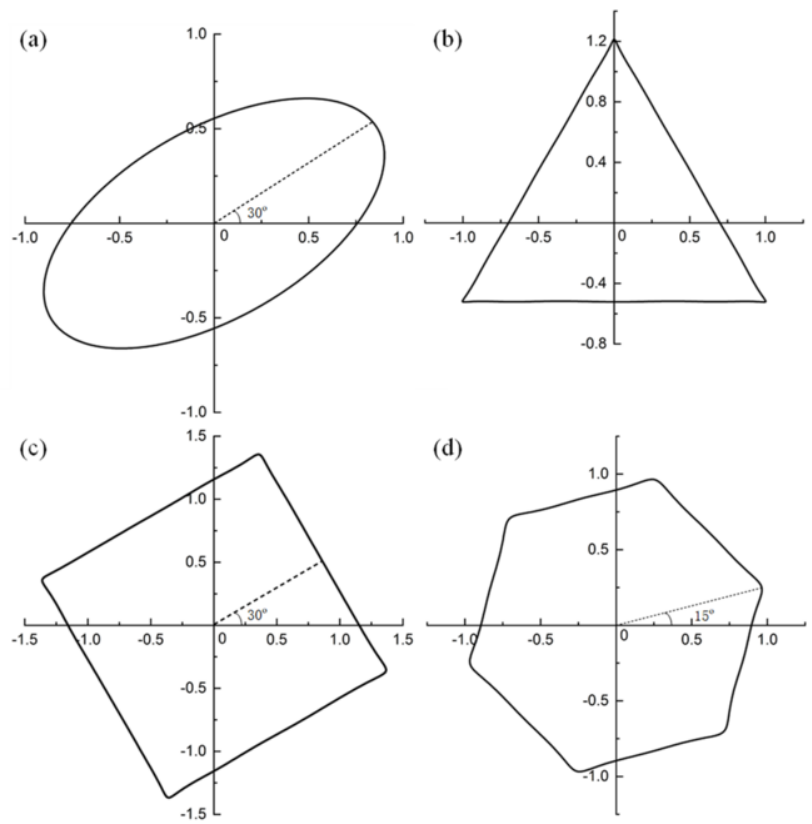

Figure 7. Combinations: (a) Oval, (b) Triangle, (c) Square, (d) Hexagon. 
Table 4. The mapping function coefficients for different geometries in figure 7.

\begin{tabular}{|c|c|c|c|c|}
\hline & Oval & Triangle & Square & Hexagon \\
\hline$c_{-1}$ & $\begin{array}{l}(0.71007,- \\
0.24055)\end{array}$ & $(0.73583,-0.41497)$ & $(1.1656,0.19546)$ & $(0.92215,-4.8705 E-2)$ \\
\hline$c_{0}$ & $\begin{array}{l}(-2.4003 \mathrm{E}- \\
4,5.4658 \mathrm{E}-4)\end{array}$ & $(-2.3953 E-4,5.7328 E-2)$ & $(6.7615 \mathrm{E}-4,-2.1993 \mathrm{E}-3)$ & $(-2.8626 \mathrm{E}-4,-8.6638 \mathrm{E}-4)$ \\
\hline$c_{1}$ & $\begin{array}{l}(4.9193 \mathrm{E}- \\
2,0.24424)\end{array}$ & (1.3953E-4,2.9350E-4) & $(1.2535 \mathrm{E}-3,-9.7808 \mathrm{E}-4)$ & $(-3.5183 \mathrm{E}-5,-6.7410 \mathrm{E}-4)$ \\
\hline$c_{2}$ & & $(0.24191,-0.14633)$ & $(2.4320 \mathrm{E}-3,-2.2312 \mathrm{E}-4)$ & $(-1.3766 \mathrm{E}-4,-5.9978 \mathrm{E}-4)$ \\
\hline$c_{3}$ & & $(-2.4540 \mathrm{E}-4,2.2411 \mathrm{E}-4)$ & $(5.3812 E-3,-0.19787)$ & $(-3.2144 \mathrm{E}-4,-6.0976 \mathrm{E}-4)$ \\
\hline$c_{4}$ & & $(-1.1524 \mathrm{E}-4,2.3328 \mathrm{E}-4)$ & $(-1.3381 \mathrm{E}-3,-2.1334 \mathrm{E}-4)$ & $(-7.0092 \mathrm{E}-4,-8.5111 \mathrm{E}-4)$ \\
\hline$c_{5}$ & & (1.6577E-2,-1.0884E-2) & $(-2.0258 E-4,-5.2033 E-4)$ & $(-1.6729 \mathrm{E}-2,6.2398 \mathrm{E}-2)$ \\
\hline$c_{6}$ & & $(1.8462 \mathrm{E}-4,4.2741 \mathrm{E}-5)$ & $(-5.5365 E-5,-1.4917 E-3)$ & $(6.2500 \mathrm{E}-4,-1.2294 \mathrm{E}-4)$ \\
\hline$c_{7}$ & & $(1.2088 E-5,-6.6912 E-5)$ & $(-2.1301 \mathrm{E}-2,2.1203 \mathrm{E}-3)$ & $(3.5856 \mathrm{E}-4,1.7406 \mathrm{E}-4)$ \\
\hline$c_{8}$ & & $(4.4258 E-3,-3.2537 E-3)$ & $(-2.9561 \mathrm{E}-5,-2.9406 \mathrm{E}-4)$ & (3.1094E-4,1.4535E-4) \\
\hline$c_{9}$ & & (1.0649E-4,3.0993E-4) & $(-1.1720 \mathrm{E}-4,4.2141 \mathrm{E}-6)$ & $(3.0898 \mathrm{E}-4,9.3731 \mathrm{E}-5)$ \\
\hline$c_{10}$ & & (2.1984E-4,3.0512E-4) & $(1.6128 \mathrm{E}-4,-2.3872 \mathrm{E}-4)$ & (1.5796E-4,-1.1818E-4) \\
\hline$c_{11}$ & & $(2.5585 E-3,-1.7658 E-3)$ & $(1.4370 \mathrm{E}-3,6.2268 \mathrm{E}-3)$ & $(-8.0822 E-3,5.4006 E-3)$ \\
\hline$c_{12}$ & & & $(-1.6262 E-4,-4.4685 E-4)$ & $(-7.5624 \mathrm{E}-5,2.8443 \mathrm{E}-4)$ \\
\hline$c_{13}$ & & & $(-6.8037 \mathrm{E}-4,-4.3629 \mathrm{E}-4)$ & $(-3.4352 E-4,2.7102 E-4)$ \\
\hline$c_{14}$ & & & $(-3.8986 \mathrm{E}-4,-1.5783 \mathrm{E}-4)$ & $(-4.1703 E-4,2.2590 E-4)$ \\
\hline$c_{15}$ & & & $(1.7334 \mathrm{E}-3,1.6694 \mathrm{E}-4)$ & $(-4.8575 \mathrm{E}-4,2.1500 \mathrm{E}-4)$ \\
\hline
\end{tabular}

\subsection{The Annular Domain Bounded by Two Closed Jordan Curves}

Let $D_{z}$ be the finite doubly-connected domain whose boundary components are shown in figure 8. The obtained mapping function coefficients are listed in table 5. If the mapping domain is symmetric with respect to $y$-axis, the $j$ th ( $j$ is even) power of $\zeta$ in $\omega_{b}(\zeta)$ is vanished. In particular, if the mapping domain exhibits four axes of symmetry (see figure $8(\mathrm{~d}))$, only mapping coefficients $c_{j}(j=-l,-(l-4), \ldots,-3,1,5, \ldots,(n-$ $4), n$ ) will appear [1]. In this case, the mapping function coefficients can be obtained by only eliminating the redundant terms in matrices $\mathbf{X}$ and $\mathbf{Y}$ in Eq. (5).

Table 5. The mapping function coefficients for different geometries in figure 8 .

\begin{tabular}{|c|c|c|c|c|}
\hline & Oval in an oval & Horse-shoe in a horse-shoe & Oval in a circle & Square in a circle \\
\hline$c_{-11}$ & & $1.1578 \mathrm{E}-3$ & & $-1.9594 \mathrm{E}-5$ \\
\hline$c_{-10}$ & & $-4.6977 \mathrm{E}-4$ & & \\
\hline$c_{-9}$ & $1.9133 \mathrm{E}-8$ & $-1.5842 \mathrm{E}-3$ & $4.0345 E-8$ & \\
\hline$c_{-8}$ & & $3.1817 E-3$ & & \\
\hline$c_{-7}$ & $2.3282 \mathrm{E}-7$ & $-7.8804 \mathrm{E}-4$ & $3.9900 \mathrm{E}-7$ & $4.0506 \mathrm{E}-4$ \\
\hline$c_{-6}$ & & $-5.3278 \mathrm{E}-3$ & & \\
\hline$c_{-5}$ & $5.9887 \mathrm{E}-7$ & $1.2420 \mathrm{E}-2$ & 3.7540E-6 & \\
\hline$c_{-4}$ & & $-4.2666 \mathrm{E}-3$ & & \\
\hline$c_{-3}$ & $3.7249 \mathrm{E}-5$ & $-1.5158 \mathrm{E}-2$ & $9.2132 \mathrm{E}-5$ & $-3.8779 \mathrm{E}-2$ \\
\hline$c_{-2}$ & & $7.4812 \mathrm{E}-2$ & & \\
\hline$c_{-1}$ & 0.29976 & -0.25328 & $7.8497 \mathrm{E}-2$ & \\
\hline$c_{0}$ & & 0.21242 & & \\
\hline$c_{1}$ & 1.4999 & 1.8835 & 1.4949 & 2.0032 \\
\hline$c_{2}$ & & $3.5679 \mathrm{E}-2$ & & \\
\hline$c_{3}$ & $4.4511 \mathrm{E}-4$ & -0.11025 & $-7.2039 E-2$ & \\
\hline$c_{4}$ & & $3.0196 \mathrm{E}-2$ & & \\
\hline$c_{5}$ & $-2.9224 \mathrm{E}-5$ & $3.2096 \mathrm{E}-3$ & $6.3666 \mathrm{E}-3$ & 3.7204E-2 \\
\hline$c_{6}$ & & $-1.2928 \mathrm{E}-2$ & & \\
\hline$c_{7}$ & $-2.1199 \mathrm{E}-5$ & 8.5990E-3 & $-1.7400 \mathrm{E}-3$ & \\
\hline$c_{8}$ & & $-1.0084 \mathrm{E}-3$ & & \\
\hline$c_{9}$ & $-2.8792 \mathrm{E}-5$ & $-7.6757 E-3$ & $-5.9515 E-4$ & $2.7238 \mathrm{E}-4$ \\
\hline$c_{10}$ & & $3.4637 \mathrm{E}-3$ & & \\
\hline$c_{11}$ & & $1.0180 \mathrm{E}-3$ & & \\
\hline
\end{tabular}




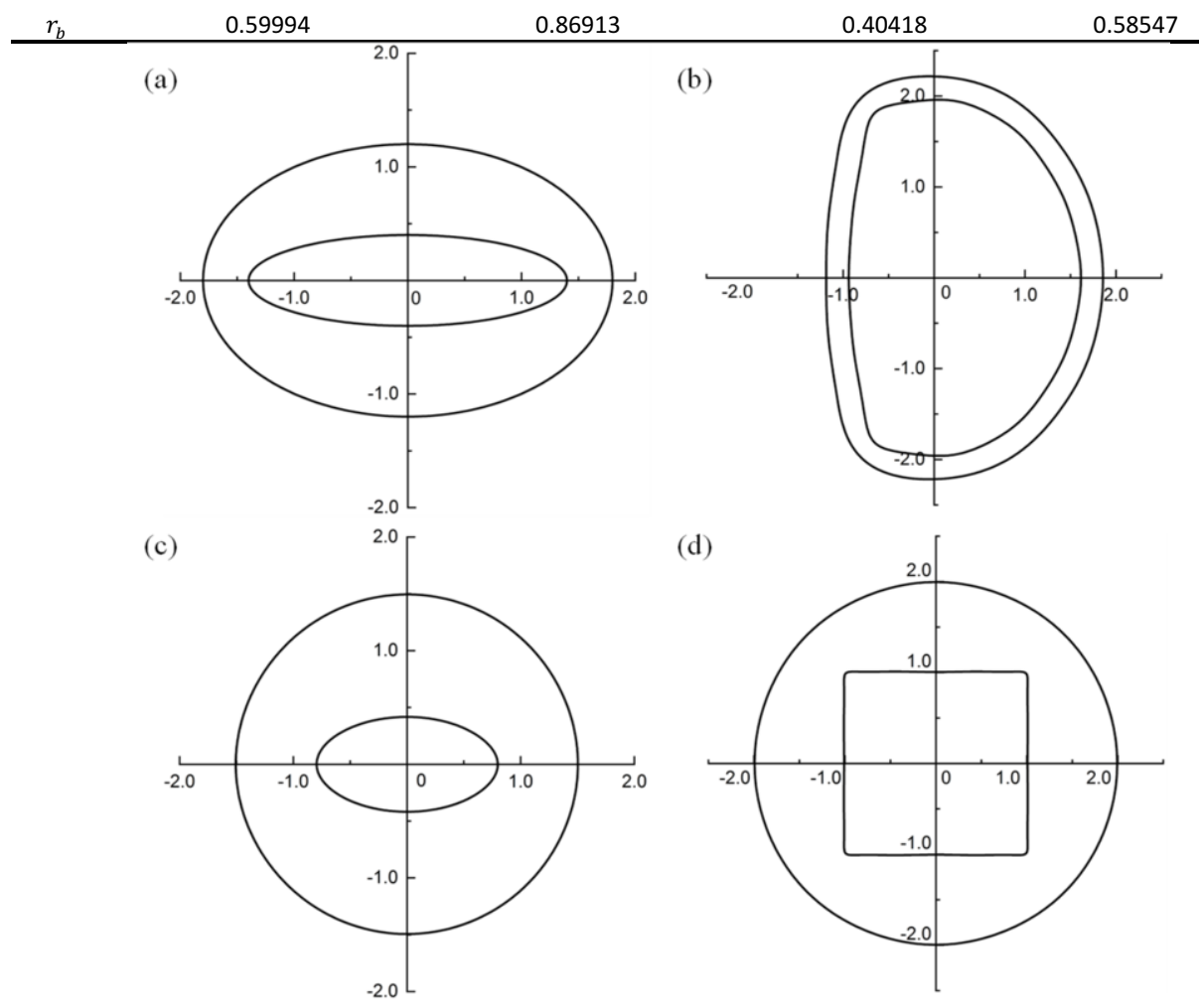

Figure 8. Combinations: (a) Oval in an oval, (b) Horse-shoe in a horse-shoe, (c) Oval in a circle, (d) Square in a circle.

In figure 8(a), the two ovals are confocal and there is an analytic solution which maps the ovals onto an annulus [18]. The value of $r_{b}$ obtained by the proposed method is 0.59994 , which is in good agreement with the analytic solution 0.6. And figure $8(\mathrm{~b})$ defines two similar horse-shoe shaped boundaries, however, there are small errors at the sharp corners which are not overcome by the method.

\subsection{The Infinite Domain Bounded by Two Closed Jordan Curves}

Let $D_{z}$ be the infinite triply-connected domain defined in Section 3.3, whose boundary are given in figure 9. The obtained mapping function coefficients are listed in table 6 . Similarly, the symmetry of the mapping domains in figure 9(a) and figure 9(b) are used.

The shapes in figure 9 are collected from the twin-tunnel problem in underground engineering, and it shows that the method proposed in this paper can better solve the insurmountable problem in reference [26]. 


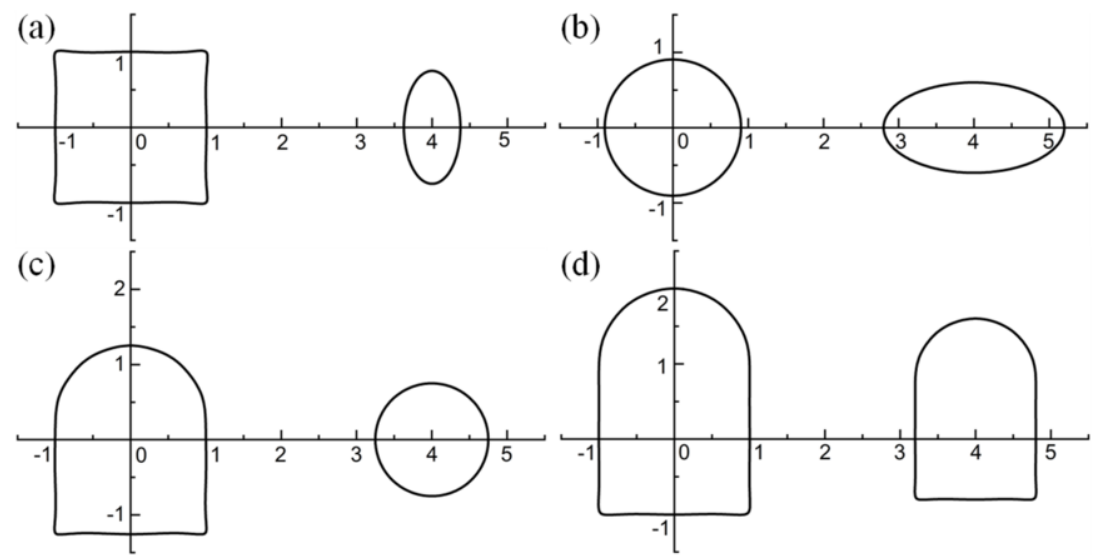

Figure 9. Combinations: (a) Square-oval, (b) Circle-oval, (c) Vertical-wall semicircle-circle, (d) Two verticalwall semicircles.

Table 6. The mapping function coefficients for different geometries in figure 9 .

\begin{tabular}{|c|c|c|c|c|}
\hline & Square-oval & Circle-oval & $\begin{array}{c}\text { Vertical-wall semicircle- } \\
\text { circle }\end{array}$ & Two vertical-wall semicircles \\
\hline$c^{\prime}$ & 1.1766 & 0.91599 & $(1.2414,4.0752 \mathrm{E}-2)$ & $(1.3667,-2.2974 \mathrm{E}-2)$ \\
\hline$c_{0}$ & $-2.8392 \mathrm{E}-2$ & 7.2359E-2 & $(-5.1291 E-4,-0.12657)$ & $(-5.9496 E-2,0.37765)$ \\
\hline$c_{1}$ & $-3.1265 E-4$ & $8.7447 \mathrm{E}-4$ & $(-0.11803,4.6651 E-3)$ & $(-0.24796,-3.7751 E-3)$ \\
\hline$c_{2}$ & $1.1553 \mathrm{E}-3$ & $1.9131 \mathrm{E}-4$ & $(-5.6592 E-3,-9.3138 E-2)$ & (3.3896E-3,-8.4205E-2) \\
\hline$c_{3}$ & -0.20101 & $7.6589 \mathrm{E}-5$ & $(-0.12179,1.2049 E-2)$ & $(-0.14129,-9.8826 E-3)$ \\
\hline$c_{4}$ & $-1.0705 \mathrm{E}-3$ & $-7.9637 E-6$ & (6.5739E-3,4.7880E-2) & $(-6.1032 E-3,5.1798 E-2)$ \\
\hline$c_{5}$ & $-2.4429 E-6$ & $-7.7048 E-5$ & $(-4.7252 E-3,4.5766 E-4)$ & $(-2.5086 \mathrm{E}-3,2.6518 \mathrm{E}-4)$ \\
\hline$c_{6}$ & $-3.2690 \mathrm{E}-4$ & $-6.1476 \mathrm{E}-6$ & (2.2059E-3,1.2196E-2) & $(-1.3625 E-3,5.2319 E-3)$ \\
\hline$c_{7}$ & $2.1192 \mathrm{E}-2$ & $-4.6018 \mathrm{E}-5$ & (8.9879E-3,-2.1027E-3) & (6.0879E-3,1.3290E-3) \\
\hline$c_{8}$ & $3.3829 E-4$ & $-5.1953 E-6$ & $(-2.1976 E-3,-7.9069 E-3)$ & $(1.4369 \mathrm{E}-3,-8.6580 \mathrm{E}-3)$ \\
\hline$c_{9}$ & $1.2122 \mathrm{E}-4$ & $-2.7879 E-5$ & $(-2.4172 \mathrm{E}-3,7.2238 \mathrm{E}-4)$ & $(-2.9966 \mathrm{E}-3,-7.8302 \mathrm{E}-4)$ \\
\hline$c_{10}$ & $-1.8914 \mathrm{E}-4$ & 4.4327E-6 & $(-1.0090 \mathrm{E}-3,-2.9616 \mathrm{E}-3)$ & $(2.3242 \mathrm{E}-4,-1.6948 \mathrm{E}-3)$ \\
\hline$c_{-1}$ & $-8.9233 E-2$ & 0.29443 & $(-2.7230 \mathrm{E}-4,4.7118 \mathrm{E}-5)$ & $(-0.15748,-1.7647 E-3)$ \\
\hline$c_{-2}$ & $5.7956 \mathrm{E}-5$ & $-1.0284 \mathrm{E}-5$ & $(3.4515 E-5,-1.8363 E-6)$ & (9.9577E-4,-4.2943E-2) \\
\hline$c_{-3}$ & $2.8368 \mathrm{E}-6$ & $-1.2274 \mathrm{E}-5$ & (-4.9347E-6,2.9369E-7) & $(-5.6714 \mathrm{E}-2,-9.6781 \mathrm{E}-4)$ \\
\hline$c_{-4}$ & 1.0194E-6 & $-2.5531 E-6$ & (1.5489E-7,9.0442E-8) & (1.7046E-4,1.6457E-2) \\
\hline$c_{-5}$ & $-1.9329 E-7$ & $-8.1659 E-5$ & (5.9346E-8,-2.4012E-7) & $(-6.4543 E-4,-3.0315 E-4)$ \\
\hline$c_{-6}$ & $-8.9968 \mathrm{E}-9$ & $-2.8390 \mathrm{E}-6$ & (2.0766E-7,1.3814E-7) & $(4.2475 \mathrm{E}-5,1.1254 \mathrm{E}-3)$ \\
\hline$c_{-7}$ & $-3.0027 E-7$ & $3.8167 E-6$ & $(-4.7644 \mathrm{E}-8,7.9099 \mathrm{E}-8)$ & $(1.0602 \mathrm{E}-3,-4.5839 \mathrm{E}-5)$ \\
\hline$c_{-8}$ & $-1.4313 E-8$ & $-2.7981 E-6$ & (3.2481E-8,-2.9219E-8) & $(4.0667 E-5,-1.1326 E-3)$ \\
\hline$c_{-9}$ & $-6.0136 \mathrm{E}-8$ & $-5.1658 E-6$ & (5.0177E-8,5.9180E-8) & $(-3.0426 \mathrm{E}-4,1.5585 \mathrm{E}-6)$ \\
\hline$c_{-10}$ & $-2.7427 \mathrm{E}-9$ & $3.6700 \mathrm{E}-7$ & $(-3.6618 \mathrm{E}-8,4.2500 \mathrm{E}-8)$ & $(1.5206 \mathrm{E}-5,-1.4109 \mathrm{E}-4)$ \\
\hline$s^{\prime}$ & 1.9521 & 2.3051 & 1.6600 & 1.2444 \\
\hline$r_{c}$ & 0.47620 & 0.98249 & 0.59678 & 0.79334 \\
\hline
\end{tabular}

\section{Conclusions}

This paper used the optimization method to present a general method for determining the conformal mapping from the unit disc onto the infinite domain exterior of a given Jordan curve, from the annulus domain onto the finite doubly-connected domain, from the exterior domain of two circles onto the exterior domain of two given Jordan curves. The method can be reduced to the construction and solution of the complex equations 
$\mathbf{Z}=\mathbf{X Y}$. The ingenuity of the method is that it introduces the least square principle to transform the problem of solving the mapping function coefficients into a problem of determining the angles on the $\zeta$-plane. Then the angles are set as the design variables of the mixed penalty function. The angles are arranged in the interval of $[0,2 \pi]$ in an increasing order, which greatly reduces the feasible region of design variables and makes it easy to evaluate the initial value of design variables.

In practice, the power series approximations can be tried if the form of the maps are unknown. The performed numerical examples show that the calculation will achieve high accuracy as long as the number of the terms of the mapping function are large enough. The solution in our paper are suitable for solving domains without symmetry axes of coordinates. Except for the applications discussed in the paper, the method may be promising in addressing more conformal mappings from circular domains to given domains.

\section{References}

[1] Schinzinger R, Laura PAA. Conformal Mapping: Methods and Applications. New York: Dover Publications Inc.; 2003.

[2] Thompson JF, Warsi ZUA, Mastin CW. Numerical grid generation: foundations and applications. Amsterdam: North-Holland; 1982.

[3] Kythe PK. Computational Conformal Mapping. Boston: Birkhuser Boston, Inc.; 1998.

[4] Trefethen LN. Numerical computation of the Schwarz-Christoffel transformation. SIAM J Sci Statist Comput. 1980; 1: 82-102.

[5] Andersson A. Schwarz-Christoffel mappings for nonpolygonal regions. SIAM J Sci Comput. 2008; 31: 94-111.

[6] Andersson A. Modified Schwarz-Christoffel mappings using approximate curve factors. J Comput Appl Math. 2009; 233: 1117-1127.

[7] Papamichael N, Kokkinos CA. Two numerical methods for the conformal mapping of simply-connected domains. Comput Method Appl M. 1981; 28: 285-307.

[8] Fornberg B. A Numerical Method for Conformal Mappings. SIAM J Sci Statist Comput. 1980; 1: 386400.

[9] Lu AZ, Wang QW. New method of determination for the mapping function of tunnel with arbitrary boundary using optimization techniques. Chin J Rock Mech Eng. 1995; 14: 269-274.

[10] Huangfu PP, Wu FQ, Guo SF, et al. A new method for calculating mapping function of external area of cavern with arbitrary shape based on searching points on boundary. Rock Soil Mech. 2011; 32: 14181424.

[11] Zhu JH, Yang JH, Shi GP, et al. Calculating method for conformal mapping from exterior of unit circle to exterior of cavern with arbitrary excavation cross-section. Rock Soil Mech. 2014; 35: 175-183.

[12] Muskhelishvili NI. Some basic problems of the mathematical theory of elasticity. Netherlands: Springer; 1977.

[13] Papamichael N, Warby MK. Pole-type singularities and the numerical conformal mapping of doublyconnected domains. J Comput Appl Math. 1984; 10: 93-106.

[14] Kress R. Inverse problems and conformal mapping. Complex Var Elliptic. 2012; 57: 301-316.

[15] Salucci M, Boulos F, Polo A, et al. Conformal Transformation Electromagnetics based on SchwarzChristoffel Mapping for the Synthesis of Doubly-Connected Metalenses. IEEE T Antenn Propag. 2019; 68: 1836-1850.

[16] Vecheslavov VV, Tolstobrova NI. Analysis-of plane electrostatic fields in doubly connected polygonal domains using conformal mappings. J Comput Phys. 1977; 24: 133-140.

[17] Papamichael N, Kokkinos CA. The Use of Singular Functions for the Approximate Conformal Mapping of Doubly-Connected Domains. SIAM J Sci Statist Comput. 1984; 5: 684-700.

[18] Symm GT. Conformal Mapping of Doubly-Connected Domains. Numer Math. 1969; 13: 448-457.

[19] Wegmann R. An iterative method for conformal mapping. J Comput Appl Math. 1986; 14: 7-18.

[20] Hakula H, Quach T, Rasila A. Conjugate function method for numerical conformal mappings. J Comput Appl Math. 2013; 237: 340-353.

[21] Mayo A. Rapid methods for the conformal mapping of multiply connected regions. J Comput Appl Math. 1986; 14: 143-153. 
[22] DeLillo TK, Elcrat AR, Pfaltzgraff JA. Schwarz-Christoffel mapping of multiply connected domains. J Anal Math. 2004; 94: 17-47.

[23] Crowdy D. The Schwarz-Christoffel mapping to bounded multiply connected polygonal domains. Proc R Soc A. 2005; 461: 2653-2678.

[24] Crowdy D. Schwarz-Christoffel mappings to unbounded multiply connected polygonal regions. Math Proc Cambridge. 2007; 142: 319-339.

[25] DeLillo TK. Schwarz-Christoffel mapping of bounded, multiply connected domains. Comput Meth Funct Th. 2006; 6: 275-300.

[26] Zeng XT, Lu AZ. Stress Solution for an Infinite Elastic Plate Containing Two Arbitrarily Shaped Holes. AIAA J. 2019; 57: 1691-1701.

[27] Mityushev V. Riemann-Hilbert Problems for Multiply Connected Domains and Circular Slit Maps. Comput Mech Funct Th. 2012; 11: 575-590.

[28] Mityushev V. Schwarz-Christoffel Formula for Multiply Connected Domains. Comput Mech Funct Th. 2012; 12: 449-463.

[29] Klonowska ME, Prosnak WJ. On an effective method for conformal mapping of multiply connected domains. Acta Mech, 1996; 119: 35-52.

[30] Klonowska ME. A pseudospectral method for the direct and the inverse thermal problem in plane, multiply connected domains. Acta Mech, 1998; 130: 55-65.

[31] Prosnak WJ, Czesnik PP. Transformation of the Vistula Lagoon into a canonical domain. Oceanologia, 2001, 43: 169-200.

[32] Savin GN. Stress concentration around holes. Oxford: Pergamon press; 1961.

[33] Zhu JJ, Xie QH, Zuo TY, et al. Complex Least Squares Adjustment to Improve Tree Height Inversion Problem in PolInSAR. J Geodesy Geoinformation Sci. 2019; 2: 1-8.

[34] Himmelblan DM. Applied nonlinear programming. London: Mc Graw-Hill Book Co; 1972. 